\title{
McCune Albright Syndrome: A Case Report
}

\section{Subhana Thapa Karki ${ }^{1}$ and Vandana Jain ${ }^{2}$}

${ }^{1}$ Department of Paediatrics, Kanti Children's Hospital, Maharajgunj, Kathmandu, Nepal

${ }^{2}$ Department of Paediatrics, All India Institute of Medical Sciences, New Delhi, India

\section{Correspondence:}

Subhana Thapa Karki

Kanti Children's Hospital,

Maharajgunj,

Kathmandu, Nepal

E-mail: subhanakarki@gmail.com

DOI: $10.3126 /$ jnps.v40i2.29099

Submitted on: $2020-05-24$

Accepted on: 2020-06-06

Acknowledgements: None

Funding: Nil

Conflict of Interest: None declared

Permission from IRB: Yes
To cite this article: Karki ST, Jain V. McCune Albright Syndrome: A Case Report. J Nepal Paediatr Soc. 2020;40(2): $134-7$

\section{ABSTRACT}

McCune Albright syndrome (MAS) is a very rare genetic disease characterised by any two of the following three findings: café au lait spots, polyosteotic fibrous dysplasia and endocrine disorders. The clinical presentation of MAS may vary depending on which of the various components of the syndrome predominate. Here, we report one case of MAS presenting with precocious puberty.

Key words: Café au lait spots; McCune-Albright syndrome; Polyosteotic fibrous dysplasia; Precocious puberty 


\section{INTRODUCTION}

McCune-Albright Syndrome (MAS) is a rare disorder defined by the triad of peripheral precocious puberty, café-au-lait skin pigmentation and fibrous dysplasia of bone. MAS should be considered in girls with recurrent formation of follicular cysts and cyclic menses. Girls presenting with premature vaginal bleeding should be carefully evaluated for features of MAS to avoid this potential mistake.

MAS is resulted by a somatic activating mutation in the GNAS1 gene leading to increased activity of the Gs alpha subunit of peptide receptors. The syndrome might influence the functioning of other hormones acting through the Gs alpha subunit (TSH, GHRH, ACTH and PTH) and may be associated with endocrinopathies such as hyperthyroidism, gigantism or acromegaly, Cushing syndrome, hyperparathyroidism and hypophosphatemic rickets.

\section{CASE REPORT}

A five and half year old girl, developmentally normal, with no significant past history presented with history of pain and deformity of both lower limbs for four months and history of one episode of per vaginal bleeding one year back. On examination, weight was $15 \mathrm{~kg}$ (just below $3^{\text {rd }}$ centile), height was $102 \mathrm{~cm}$ (just below $3^{\text {rd }}$ centile) with US: LS ratio 1.9:1. Her pubertal development was Tanner stage of B2/PH1. Genu valgus deformity and multiple café au lait spots were present. Systemic examination findings were normal. All the investigations including haemogram, liver function tests, renal function tests, thyroid function tests, uric acid, calcium and phosphorus were normal except alkaline phosphatase (ALP)-2951 IU/L and PTH- 87.4 pg/ $\mathrm{ml}$ ( $\mathrm{N}$ 14-72) which were high and low LH and FSH with high estradiol.

Ultrasonography findings of neck showed normal thyroid and parathyroid glands and USG of abdomen and pelvis showed uterus of size $4.3 \mathrm{x}$ $1.48 \mathrm{~cm}$ (Expected length $2.83 \mathrm{~cm}$ ), right ovary-1.34 x $0.7 \mathrm{~cm}, 1 \mathrm{t}$ ovary- $2.3 \times 1.35 \mathrm{~cm}$ (Expected volume- $0.51 \mathrm{~cm}^{3}$ ) with follicular appearance. Skeletal survey revealed vertical metaphyseal striations in long bone with lytic sclerotic lesion in femoral head with coxa vera, and sclerotic lesion in small bones of hand and irregularity of lower end of ulna.

With the possible diagnosis of MAS, further investigations were done which showed normal TmP/GFR- $4.2 \mathrm{mg} / \mathrm{dl}(\mathrm{N}-2.8$ - 4.4) and normal blood glucose, serum prolactin and cortisol level. Bone scan showed diffusely increased tracer uptake of moderate intensity in skull (parieto - occipital), left ribs laterally, entire left humerus and left lower limb. After four months, height increased by 4.6 $\mathrm{cm}$, SMR progressed to B3-4, PH1. Radiography of all four limbs showed under tubulation with widening and sclerosis of left carpal, metacarpal, left tarsal, metatarsal and phalanges with loss of cortico-medullary differentiation, metaphyseal striations. Bone biopsy confirmed fibrous dysplasia.

Treatment was started with vitamin D and calcium supplements, anastrazole $1 \mathrm{mg}$ once daily in view of precocious puberty and bisphosphonate therapy with alendronate $5 \mathrm{mg}$ BD. After two months, her breasts size regressed to stage 2 and serum $\mathrm{Ca} / \mathrm{P}$ became normal and ALP was decreased than before and estradiol was undetectable. On subsequent follow up, ALP was persistently high though calcium, phosphorus, thyroid function tests, E2 and PTH were normal, there was no vaginal bleeding but pain and limb deformity was persistent. DEXA scan showed lumbar spine $\mathrm{T}$ score of -3.8 and whole body $\mathrm{T}$ score -5.8. Phosphate

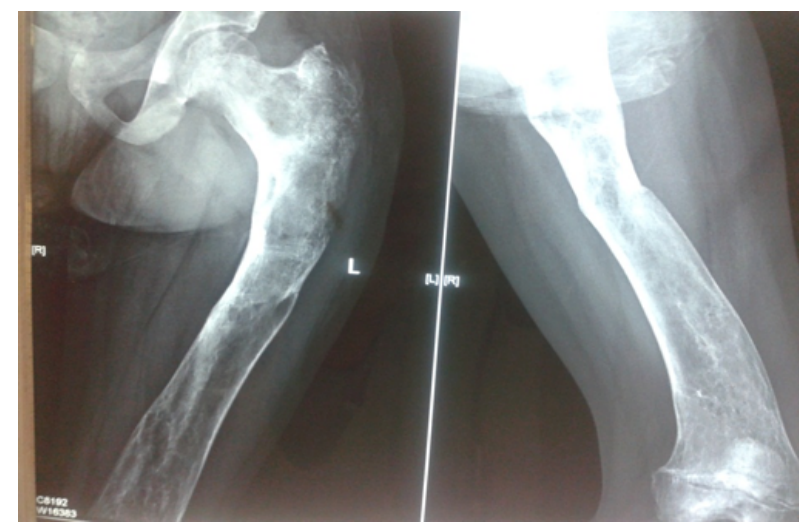

Figure 1. Ground glass appearance of left femur with fracture 
supplementation was also given from the age of eight years with frequent monitoring of calcium, phosphorus and ALP.

Later on, her bony pain regressed but the deformity was persistent and she gained normal hormonal study. Despite continuing treatment, she had fracture of left femur (Fig 1) and injection arachitol was added weekly for two months.

At 11 years, breakthrough bleeding occurred. Her height-increased to $129.8 \mathrm{~cm}$ ( $3^{\text {rd }}$ centile) and SMRB4. Uterus size became $6.7 \times 2.4 \times 2.6 \mathrm{~cm}$, right ovary - 0.8 and left ovary - 2.6, normal calcium, phosphorus with little high ALP. With this, anastrazole was stopped in view of attainment of menarche but alendronate, phosphate, vitamin D supplements were continued. At 12 and half years, her height $(131 \mathrm{~cm})$ remained static from last follow up with regular menstruation, normal TFT and bone age of 13.5 years. Thyroxine was added along with alendronate, phosphate, and vitamin D.

\section{DISCUSSION}

MAS was defined by the triad of polyostotic fibrous dysplasia of bone (FD), café-au-lait skin pigmentation, and precocious puberty (PP). ${ }^{1}$ It was later recognised that other endocrinopathies, including hyperthyroidism, growth hormone $(\mathrm{GH})$ excess, renal phosphate wasting with or without rickets/osteomalacia also occurred with this disorder. ${ }^{2-4}$ Therefore, a more clinically relevant definition of MAS, broader than the original triad of FD, PP and café-au-lait is: MAS = FD plus at least one of the typical hyper functioning endocrinopathies and/or café-au-lait spots, with almost any combination possible. ${ }^{5}$

Plain radiographs are often sufficient to make the diagnosis of FD which is a pathognomic sign. In general, lesions in the long bones have a "lytic" appearance, usually arise in the medullary cavity and expand outward replacing normal bone, which results in thinning of the cortex. It is usually the metaphysis and/or the diaphysis that are involved, with sparing of the epiphysis. The skull base and proximal femur are the sites most commonly involved, though it can involve any bone. ${ }^{6-8}$

Genetic testing is possible, but is not routinely available. Management of precocious puberty in a girl with MAS consists initially of observation. In girls with a progressive form of precocious puberty, pharmacologic intervention is recommended in order to prevent early epiphyseal fusion and augmenting adult height. The most common approach employs combination therapy in the form of an androgen receptor blocker such as spironolactone, flutamide or cyproterone acetate along with a compound that interferes with sex steroid synthesis such as ketoconazole or an aromatase inhibitor. ${ }^{9}$

Corrective surgery can be performed for bone dysplasia. Recently, bisphosphonate therapy has shown promise for fibrous dysplasia patients as it helps to diminish pain, prevents fracture and leads to partial resolution of fibrous dysplasia lesions. ${ }^{10}$

\section{CONCLUSIONS}

MAS is diagnosed from clinical features. In children with fibrous dysplasia, MAS should be a differential diagnosis to be considered. Management includes pharmacological therapy for precocious puberty mostly for girls and management of endocrinopathies.

\section{REFERENCES}

1. Claudia E Dumitrescu, Michael T Collins: McCune-Albright syndrome. Orphanet J Rare Dis. 2008,3:12. DOI: 10.1186/1750-1172-3-12. 
2. Mastorakos G, Mitsiades NS, Doufas AG, Koutras DA: Hyperthyroidism in McCune-Albright syndrome with a review of thyroid abnormalities sixty years after the first report. Thyroid. 1997;7:433-39. DOI: 10.1089/thy. 1997.7.433.

3. Sherman SI, Ladenson PW: Octreotide therapy of growth hormone excess in the McCune-Albright syndrome. J Endocrinol Invest.1992;15:185-90. DOI: 10.1007/BF03348702.

4. Akintoye SO, Chebli C, Booher S, Feuillan P, Kushner H, Leroith D, et al. Characterisation of gsp-mediated growth hormone excess in the context of McCune-Albright syndrome. J Clin Endocrinol Metab. 2002;87(11):5104-12. DOI: $10.1210 /$ jc.2001-012022.

5. Collins MT: Spectrum and natural history of fibrous dysplasia of bone. J Bone Miner Res. 2006;21(2):99-104. DOI: $10.1359 /$ jbmr.06s219.

6. Dorfman HD, Czerniak B: Fibroosseous Lesions. In Bone Tumors Edited by: Dorfman HD, Czerniak B. St. Louis, MO: Mosby. 1998:441-91.

7. Ippolito E, Bray EW, Corsi A, De Maio F, Exner UG, Robey PG, et al. Natural history and treatment of fibrous dysplasia of bone: a multicenter clinicopathologic study promoted by the European Pediatric Orthopaedic Society. J Pediatr Orthop B. 2003;12(3):155-77. DOI:10.1097/01.bpb.0000064021.41829.94.

8. Kelly MH, Brillante B, Collins MT. Pain in fibrous dysplasia of bone: age-related changes and the anatomical distribution of skeletal lesions. Osteoporosis Int. 2008;19(1):57-63. DOI: 10.1007/s00198-007-0425-x.

9. Galland F, Kamenicky P, Affres H, Reznik Y, Pontvert D, Le BoucY et al. McCune-Albright syndrome and acromegaly: effects of hypothalamo pituitary radiotherapy and/or pegvisomant in somatostatin analog-resistant patients. J Clin Endocrinol Metab. 2006;91:4957-61. DOI:10.1210/jc.2006-0561.

10. Lane JM, Khan SN, O'Connor WJ, Nydick M, Hommen JP, Schneider R, et al. Bisphosphonate therapy in fibrous dysplasia. Clin Orthop Relat Res. 2001;382:6-12. DOI: 10.1097/00003086-200101000-00003. 